\title{
Plantaciones nativas o exóticas: Reflexiones sobre los impactos ambientales en Chile
}

\author{
Álvaro Promis \\ Departamento de Silvicultura y Conservación de la Naturaleza, Facultad de Ciencias Forestales y de la Conservación de la \\ Naturaleza, Universidad de Chile. Santiago, Chile.
}

\begin{abstract}
Resumen. En este trabajo se plantea la necesidad de reorientar y diversificar la forestación, reforestación, rehabilitación o restauración con especies nativas a fin de recuperar bosques y ecosistemas más cerca de su estado natural. Esta necesidad se fundamenta en los cambios en los patrones de disturbio a nivel global, en la necesidad de promover acciones de adaptación y mitigación al cambio climático, en la conservación de especies en categoría de amenaza y en las demandas de grupos de investigadores y diversas organizaciones de la sociedad civil. Para ello, se incluyen antecedentes de las modificaciones ambientales que se produjeron al establecer plantaciones de especies arbóreas exóticas en áreas de bosque nativo. El objetivo de este debate es fomentar proyectos de forestación, reforestación y restauración que se basen en especies arbóreas nativas.

[Palabras clave: árboles nativos, forestación, reforestación, rehabilitación, restauración, plantación cerca de lo natural]

ABSTRACT. Native or exotic plantations: Thoughts about the environmental impacts in Chile. This work presents the need to reorient and diversify afforestation, reforestation, rehabilitation and/or restoration through the use of native species, to recover forests and ecosystems closer to their natural state. This need is based on changes to the global disturbance patterns, the necessity to develop adaptation and mitigation actions to climate change, the conservation of threatened species and the demands of civil society organizations and researchers. For that purpose, background information is presented, regarding environmental changes produced by the establishment of plantations of exotic tree species respect to the native forest, with the purpose of promoting afforestation, reforestation and restoration projects through the use of native tree species.
\end{abstract}

[Keywords: native tree species, afforestation, reforestation, rehabilitation, restoration, close-to-natural tree plantation]

\section{INTRODUCCIÓN}

En el pasado, las categorías de uso de suelo más extensas en Chile eran los bosques nativos y el matorral. Potencialmente, el bosque nativo alcanzaba a cubrir $38.3 \%$ de la superficie de Chile continental (28.5 millones de ha) y el matorral 34.5\% (25.8 millones ha) (Luebert and Pliscoff 2017). En la actualidad, a lo largo de Chile continental existen 14.4 millones de ha de bosques nativos, 3.1 millones de ha de plantaciones forestales y $170 \mathrm{mil}$ ha de bosques mixtos de especies nativas y exóticas (CONAF 2017a). Esto quiere decir que la presencia de bosque nativo habría disminuido a la mitad de la que potencialmente existía. En particular, en la ecorregión de los bosques valdivianos lluviosos también se estimó una disminución de la cobertura del bosque nativo a casi la mitad de la existente hacia 1550 (Lara et al. 2012). Las principales causas de cambio han sido el reemplazo de bosque nativo a pradera y matorral, el cambio a uso agrícola y el desarrollo de plantaciones forestales (Lara et al. 2012).

Editor asociado: Guillermo Martínez Pastur 两 alvaro.promis@gmail.com
La preocupación del Estado chileno por reforestar y recuperar suelos degradados se empieza a materializar hacia fines del siglo XIX y comienzos del siglo XX, considerando el establecimiento de plantaciones forestales con la introducción de especies exóticas (Camus 2006). La política de forestación comenzó por el año 1931, y se intensificó luego de que se implementó el Decreto Ley 701 (DL701) de 1974 y la Ley 19.561 de 1998, que renovó el DL701 y que duró hasta el 2012 (Reyes et al. 2014). El DL701 entregó subsidios que cubrían entre el 75 y el $90 \%$ de los costos de establecimiento y manejo de las forestaciones, además de beneficios tributarios. Se estima que la inversión del Estado fue de 474 millones de dólares durante los años 1976 y 2010 ( 13.5 millones de dólares anuales); con lo que se bonificó una superficie superior a 1.4 millones de ha (Cabaña Chávez 2011). Esta política forestal llevó a que el sector forestal represente un $2.1 \%$ del producto interno bruto de Chile, con exportaciones que ascienden a 6838 millones de dólares al año, debido en su mayoría a la industria de plantaciones con 
especies exóticas (INFOR 2019). No obstante, este éxito en crecimiento macroeconómico fue puesto en duda por las externalidades negativas que dejó el sector forestal, tanto en el ámbito social como en el ambiental (Parra 2019).

Es así como desde un punto de vista social, aunque el sector forestal genera cerca de 114 mil empleos (INFOR 2019), las comunas con mayor pobreza del país corresponden a aquellas con mayor proporción de plantaciones forestales (Reyes et al. 2014). Al mismo tiempo, desde un punto de vista ambiental, mientras se mantenía en ejecución el DL701 (alrededor de los años 1990), la institucionalidad forestal nacional planteaba que los beneficios del establecimiento de las plantaciones forestales de especies exóticas, en especial de Pinus radiata D. Don, era mayor respecto a los impactos negativos, tales como el efecto sobre la biodiversidad y las cuencas hidrográficas (Susaeta and Benedetti 1990; Camus 2006). Sin embargo, en ambas dimensiones (social y ambiental) hay profundas críticas que se fueron relevando con el tiempo (Reyes et al. 2014; Astorga and Burschel 2019).

Las plantaciones forestales se realizaron sobre todo con especies arbóreas exóticas y de rápido crecimiento, tales como $P$. radiata y especies del género Eucalyptus (Salas et al. 2016). En un principio, estas plantaciones se llevaron a cabo para solucionar el grave problema de erosión y degradación de suelos que se presentaba en Chile debido a la deforestación e histórica habilitación de terrenos para la agricultura, el abastecimiento de leña para generar energía y de madera para la construcción (Camus 2006; Prado 2015). Durante el período comprendido entre los años 1986 y 2001, el aumento de la superficie de las plantaciones de especies exóticas se debió principalmente al reemplazo del bosque nativo, patrón que fue disminuyendo hacia 2011 (Heilmayr et al. 2016). La principal función de las plantaciones ha sido la de producción comercial, y sus objetivos más importantes fueron el abastecimiento de madera y fibra para los complejos industriales, la maximización del valor presente neto de la inversión (Donoso and Otero 2005) y la maximización de la renta del suelo (Reyes et al. 2014). No obstante, a mediados del siglo pasado también se establecieron como medida de conservación de suelos, a fin de controlar la erosión y recuperar la capacidad de producción (Camus 2006).
Hoy, en diferentes acuerdos internacionales, Chile se comprometió a restaurar el 15\% de sus ecosistemas degradados para el año 2020, reforestar al menos $100 \mathrm{mil}$ ha para el año 2030, y forestar $500 \mathrm{mil}$ ha en terrenos de aptitud preferentemente forestal e incorporar a procesos de restauración otra superficie similar en áreas prioritarias para el año 2035 (Bannister et al. 2018). A estas cifras se le deben agregar la recuperación de 284 mil ha de plantaciones forestales y 105 mil ha de bosques nativos quemados durante los incendios de la temporada verano 20162017 (CONAF 2017b). Aunque la opinión pública, las organizaciones de la sociedad civil y grupos de investigadores a nivel nacional mostraron interés por recuperar, reforestar, rehabilitar o restaurar estas áreas con especies arbóreas nativas (Bannister et al. 2018), queda la duda de la posición final que tomará y promoverá el Estado a través de sus organismos sectoriales. En especial, cuando en Chile se plantea entregar subsidios del orden de los 37.5 millones de dólares anuales para el fomento de forestación, reforestación y restauración forestal por un período de 20 años (Guerra Martínez 2019).

Entonces, ante estos escenarios en los que se sigue manteniendo la incertidumbre sobre el efecto ambiental de las plantaciones forestales con especies arbóreas exóticas y la posibilidad de tener un nuevo fomento a la forestación - a través de la entrega de subsidios-, esta opinión presenta antecedentes sobre modificaciones ambientales producidas por el establecimiento de estas plantaciones respecto al bosque nativo. El objetivo del trabajo es fomentar proyectos de forestación y restauración a través de especies arbóreas nativas.

\section{EVIDENCIAS DE CAMBIOS AMBIENTALES}

Los bosques nativos y las plantaciones forestales proveen múltiples beneficios para la humanidad: 1) bienes como madera, alimento, leña, forraje, recursos ornamentales y medicinales, y 2) servicios tales como secuestro de carbono, regulación de suelo y agua, hábitat para diferentes especies de flora y fauna, y recreación (Groot and van der Meer 2010). Sin embargo, salvo por la mayor rapidez para generar materia prima (madera y fibra) y recursos energéticos (leña y biocombustibles) por parte de las plantaciones respecto a los bosques nativos, prácticamente todos los servicios se ven 
reducidos a las primeras. Esto incluye los servicios de provisión (i.e., recursos genéticos, medicinas naturales, productos farmacéuticos y bioquímicos, recursos ornamentales), los de regulación (i.e., calidad del aire, clima, calidad y cantidad de agua, prevención de erosión, control biológico y polinización), los servicios culturales (i.e., estética, turismo recreacional, patrimonio cultural y de identidad, inspiración, información espiritual y religiosa, educacional, científico) y los de soporte (i.e., hábitat para especies de flora y fauna y mantención de biodiversidad) (Groot and van der Meer 2010).

Sin duda, una plantación genera más servicios que una pradera artificial o un cultivo agrícola abandonado, sobrexplotado o erosionado, tales como aquellas superficies donde se establecieron inicialmente las plantaciones forestales a principios y mediados del siglo pasado (Camus 2006). Pero la corta y la quema de bosques nativos para establecer campos agrícolas, ganaderos o plantaciones forestales de especies exóticas de rápido crecimiento implica cambios dramáticos en los atributos propios de un ecosistema forestal, es decir en la estructura, la composición de especies y las funciones y procesos ecológicos. Los bosques nativos presentan árboles con varias edades (estructura multietánea), alta diversidad de hábitats y especies, así como ciclos de nutrientes e hidrológicos más balanceados. Por el contrario, una plantación forestal de especies exóticas es monoespecífica (una sola especie), presenta una alta densidad de árboles con solo una clase de edad (plantaciones coetáneas) y se maneja en rotaciones cortas o relativamente cortas, para que siempre esté creciendo a tasas altas, lo que influye de manera directa en que los procesos y funciones sean acelerados, como por ejemplo elevadas tasas de consumo de agua y nutrientes, y que la provisión de diversos bienes y servicios sea mermada (Donoso and Otero 2005).

El cambio en los procesos ecológicos en las plantaciones con respecto a los bosques nativos es sustancial. Acá me referiré a cuatro de estos aspectos: microclima, suelo y ciclo de nutrientes; biodiversidad; ciclo hidrológico y secuestro y almacenamiento de carbono. Aunque depende del objetivo de la plantación y el manejo que se efectúe, las altas densidades de árboles y coberturas de copa en las plantaciones forestales de coníferas exóticas en Chile producen una intercepción elevada de la luz que llega al piso del bosque, lo que luego influye en una reducción de la temperatura del suelo en comparación con bosques de especies latifoliadas (Donoso et al. 2014). Esta menor temperatura del suelo disminuye la tasa de descomposición de la materia orgánica que se encuentra en el suelo (Thiers et al. 2014). En general, la hojarasca de las especies de coníferas es más ácida y pobre en nitrógeno que la de especies latifoliadas y caducifolias, lo que también influye en su baja tasa de descomposición. Esto, en las plantaciones, puede producir una disminución de los procesos de mineralización, de la actividad biológica y de retorno de nutrientes al suelo (Lusk et al. 2001; Thiers et al. 2014). En el largo plazo puede ocurrir una mayor acidez del suelo (Schlatter and Otero 1995; Rivas et al. 2009; Thiers et al. 2014) y del agua que es capaz de infiltrar (Oyarzún et al. 2005) al cambiar la vegetación dominante del bosque nativo de latifoliadas a plantación de $P$. radiata, lo cual puede afectar su fertilidad (Schlatter and Otero 1995). A lo anterior se puede agregar una disminución de la productividad del sitio por la alta demanda de nutrientes del suelo, por las altas tasas de crecimiento de los árboles y las rotaciones cortas con las que se manejan estas plantaciones (Gerding 2009).

El tema de la biodiversidad en plantaciones forestales respecto a los bosques nativos ha sido ampliamente discutido, y la información disponible es contrastante (Brockerhoff et al. 2008). Plantaciones forestales monoespecíficas y monoestratificadas influyen sobre la disminución de la biodiversidad en su interior (Braun et al. 2017). Sin embargo, existen evidencias a nivel global que indican mejoras de la biodiversidad cuando hay un adecuado manejo de la plantación forestal (e.g., un período más largo de rotación, un mosaico de rodales con diferentes edades, plantaciones mixtas, etc.) y cuando hay influencia de bosques o vegetación nativa cerca de la plantación (Lindenmayer et al. 2003; Braun et al. 2017). En Chile central, además, la riqueza de especies de plantas nativas y endémicas estaría amenazada por la presencia de las grandes y continuas superficies con plantaciones forestales que rodean los pequeños fragmentos de bosque nativo (Braun et al. 2017). En la Patagonia Chilena (región de Aysén) también se produce una disminución en la riqueza de especies de plantas al comparar plantaciones respecto a bosque nativo, especialmente de flora vascular nativa (Braun et al. 2017). Por otro lado, la diversidad de animales también se 
ve disminuida por las plantaciones forestales. Para avanzar en la búsqueda de soluciones se propuso mantener un sotobosque continuo $\mathrm{y}$ abundante en las plantaciones forestales, rotaciones más largas y un paisaje en el que se exista un mosaico de rodales con amplias clases de edades (Lindenmayer et al. 2003; Tomasevic and Estades 2008), opciones que deberían ser implementadas y monitoreadas.

Las plantaciones forestales de especies exóticas de rápido crecimiento también reducen el suministro de agua (ÁlvarezGarretón et al. 2019) al disminuir los caudales de los meses secos en las cuencas donde se encuentran establecidas (Little et al. 2009), el balance de agua (Huber et al. 2008) y el aumento del transporte de sedimentos (Oyarzún et al. 2011). Para disminuir este efecto se sugiere trabajar con rotaciones más largas (Scott and Prinsloo 2008) y una efectiva protección de las quebradas con bosques de especies nativas (Little et al. 2015).

El secuestro de carbono en plantaciones forestales comerciales también se ha puesto en duda, debido especialmente a que el carbono almacenado en suelo y plantas es $28 \%$ menor en plantaciones respecto a bosques nativos (Liao et al. 2010) y a que el carbono secuestrado se libera rápidamente después de la cosecha de los árboles, la descomposición de los restos de la plantación y de los productos obtenidos de la madera (en gran parte fibra para celulosa y producción de papel) (Lewis et al. 2019). Por un lado, en el entendido de la ausencia de disturbios de gran escala se predice un incremento del almacenamiento anual de carbono en fases de desarrollo tempranas de un bosque (i.e., fase de acumulación sensu Bormann and Likens [1979]), como son las plantaciones forestales de especies exóticas, y disminuciones hacia fases tardías, maduras o adultas (i.e., fase de equilibrio dinámico sensu Bormann and Likens [1979]) cuando la tasa de almacenamiento de carbono en el tiempo llega a ser nulo (Odum 1969). Sin embargo, hoy, esta teoría se puso en duda al considerar la evidencia de que, con el tiempo, los árboles siguen acumulando biomasa y que los bosques adultos pueden continuar almacenando carbono en la biomasa viva y en la hojarasca a través de la lenta descomposición de la materia orgánica y el almacenamiento del carbono en el suelo (Luyssaert et al. 2008; Schulze et al. 2009; Curtis and Gough 2018). Además, en la medida que el bosque entra en fases de desarrollo más tardías, la presencia de disturbios de pequeña y mediana escala (i.e., claros de dosel) se hacen más frecuentes, lo que permite aumentar la absorción de carbono por los árboles liberados y compensar la pérdida de carbono secuestrado por la descomposición (Curtis and Gough 2018). De esta manera, se estima que si se recuperan bosques nativos (con árboles que alcanzan edades adultas y rotaciones más largas) el secuestro de carbono podría llegar a ser 40 veces más grande que la obtenida por una plantación forestal comercial, donde los árboles no alcanzan edades adultas y se manejan con rotaciones cortas (Lewis et al. 2019). Por otro lado, los productos obtenidos de la madera tienen diferentes tiempos de duración y de secuestro de carbón, en promedio de 2 años para productos derivados de la celulosa (i.e., papel), 25 años para productos basados en paneles y 35 años para productos basados en madera aserrada (IPCC 2014). En Chile, en su mayoría, la madera que proviene de las plantaciones se transforma en celulosa, desde donde se generan productos de corta duración; mientras que la de bosque nativo, en madera aserrada (INFOR 2019), que posee mayor duración y capacidad de mantener el carbono almacenado. En consecuencia, una plantación con especies nativas debería generar productos basados en madera de mayor duración, como son muebles y materiales de construcción.

Según FAO (2011), un bosque se encuentra degradado una vez que disminuyó su capacidad de entregar bienes y servicios, a través de la pérdida de funciones ecosistémicas, por los cambios en la composición de especies y de su estructura (Vásquez-Grandón et al. 2018). Teniendo en cuenta esa definición, una plantación forestal de especies exóticas se debería considerar como un estado de degradación de un bosque nativo. En particular, cuando la plantación fue establecida en sitios que antes poseían bosques nativos.

\section{¿POR Qué Plantar esPecies ARbóREAS NATIVAS?}

Por un lado, la flora nativa de Chileevolucionó de manera aislada por varios millones de años; cerca del 39\% de las especies están clasificadas como endémicas (sólo se encuentran en Chile) (Rodríguez et al. 2018). En este sentido, el 44\% de las especies de árboles y árboles pequeños (51 especies) que se encuentran distribuidas de manera natural en el continente son endémicas de Chile (Rodríguez et al. 2018). De estos árboles y árboles pequeños de Chile 
continental, el 25\% de las especies nativas (30 especies considerando las endémicas) se encuentra clasificada en una alta categoría de amenaza (Vulnerable, En Peligro o en Peligro Crítico). Considerando sólo a las endémicas, el 33\% (17 especies) se encuentra amenazado en su existencia. Por lo tanto, para proteger nuestro patrimonio natural es muy necesario continuar desarrollando planes de conservación de estas especies, en especial con proyectos desarrollados in situ.

Por otro lado, si en Chile el desarrollo de las plantaciones forestales industriales con especies exóticas, la institucionalidad forestal y la creación de empresas forestales, que está vigente hasta estos días, comenzó a implementarse desde hace casi un siglo (Donoso and Otero 2005; Camus 2006; Reyes et al. 2014), entonces surgen preguntas como: 1) ¿Por qué no se puede proyectar la provisión de múltiples servicios de plantaciones forestales con especies nativas a partir de la inversión que el Estado de Chile estima realizar con el nuevo subsidio en fomento forestal (37.5 millones de dólares al año)?, 2) ¿Debe ser el rol del Estado la maximización de la renta del suelo y de la inversión?

En tiempos en que, a nivel internacional, se está informando sobre cambios en los patrones de disturbios de ecosistemas de bosques (e.g., mayor frecuencia, intensidad y severidad de incendios), se promueve un enfoque de fomento a la resiliencia de los bosques; esto permite su recuperación y adaptación a los cambios (Seidl et al. 2017), algo que se puede sustentar usando árboles de especies nativas (Bozzano et al. 2014). En Chile también se ha reportado un incremento en la ocurrencia y severidad de incendios forestales (Úbeda and Sarricolea 2016; González et al. 2020); 99\% de ellos están originados por el ser humano y sus actividades, por la ocupación de la zona de interfaz urbano-rural, por el aumento de las temperaturas y el cambio en el uso de suelo y su cobertura, por plantaciones forestales de especies exóticas y matorral, que influye sobre el tipo, cantidad e inflamabilidad del material (González et al. 2020). Para ello se propone disminuir la presencia de especies exóticas invasoras, lo que favorece la ocurrencia y propagación de incendios, la restauración de bosques con especies nativas y generar políticas de ordenamiento territorial, que promuevan paisajes más heterogéneos (Astorga and Burschel 2019; González et al. 2020). Además, las especies nativas pueden ser preferidas por las comunidades locales
(Cerda et al. 2019), cuyos conocimientos etnobotánicos y etnoecológicos de especies nativas pueden facilitar el uso exitoso de estas especies en procesos de restauración (Bozzano et al. 2014).

El desarrollo y la implementación de planes para la forestación, reforestación, rehabilitación, recuperación o restauración del bosque nativo son necesarios para que se pueda recuperar la entrega de bienes y servicios de estos bosques, para mantener la diversidad de especies endémicas y nativas y mejorar el estado de conservación de ellas, y para mantener los procesos y funciones ecológicas que ellos sustentan. Esto es especialmente relevante en tiempos en que ha aumentado el conocimiento sobre ecofisiología (autoecología) y silvicultura de las especies arbóreas nativas más frecuentes (Donoso 2016).

Por lo tanto, en virtud de la incertidumbre generada por el cambio climático y el cambio en los patrones de disturbios forestales, el Estado y sus organismos sectoriales deberían fomentar el desarrollo de ecosistemas forestales más cerca de su condición natural. En particular cuando Chile quiere comprometer la reducción de emisiones de dióxido de carbono y adaptarse al cambio climático, a través de la captura de estas emisiones en plantaciones forestales, considerando la forestación y reforestación de vastas superficies (Bannister et al. 2018).

Por todo lo planteado anteriormente, se debe trabajar en reorientar y diversificar la matriz de las nuevas plantaciones forestales a través del uso de árboles nativos, a fin de recuperar bosques y sus ecosistemas asociados hacia una condición más cercana a la natural en la que se puedan proveer beneficios múltiples para las comunidades. Sin embargo, al mismo tiempo, se debe avanzar en el establecimiento de políticas que busquen la integración de la mayor provisión de bienes y servicios en la planificación y desarrollo nacional, considerando la asignación de un valor de mercado y la promoción de herramientas políticas para el pago de dichos bienes y servicios, comerciales y no comerciales (Cerda et al. 2014; Rodríguez García et al. 2016). No obstante, se deberían comenzar a evaluar las variaciones ambientales y la provisión de bienes y servicios que se producen en las plantaciones forestales con especies arbóreas nativas ( 6500 ha), en su mayoría realizadas con especies del género Nothofagus (Salas et al. 
2016), respecto al bosque nativo, para validar o discutir la información científica relevada en esta reflexión.
Agradecimientos. Se agradece la lectura crítica, correcciones y observaciones realizadas por P. J. Donoso.

\section{REFERENCIAS}

Álvarez-Garretón, C., A. Lara, J. P. Boisier, and M. Galleguillos. 2019. The impacts of native forests and forest plantations on water supply in Chile. Forests 10:473. https://doi.org/10.3390/f10060473.

Astorga, L., and H. Burschel (eds.). 2019. Chile necesita un nuevo modelo forestal: Ante los desafíos climáticos, sociales y ambientales. LOM Ediciones, Santiago, Chile.

Bannister, J. R., R. Vargas-Gaete, J. F. Ovalle, M. Acevedo, A. Fuentes-Ramírez, P. J. Donoso, A. Promis, and C. SmithRamírez. 2018. Major bottlenecks for the restoration of natural forests in Chile. Restoration Ecology 26:1039-1044. https://doi.org/10.1111/rec.12880.

Bormann, F. H., and G. E. Likens. 1979. Pattern and process in a forested ecosystem. Springer-Verlag, New York, Estados Unidos. https: / / doi.org/10.1007/978-1-4612-6232-9.

Bozzano, M., R. Jalonen, E. Thomas, D. Boshier, L. Gallo, S. Cavers, S. Bordács, P. Smith, and J. Loo (eds.). 2014. Genetic considerations in ecosystem restoration using native tree species. State of the World's Forest Genetic Resources Thematic Study. FAO and Biodiversity International, Roma, Italia.

Braun, A. Ch., D. Troeger, R. García, M. Aguayo, R. Barra, and J. Vogt. 2017. Assessing the impact of plantation forestry on plant biodiversity. A comparison of sites in Central Chile and Chilean Patagonia. Glob Ecol Conserv 10:159-172. https://doi.org/10.1016/j.gecco.2017.03.006.

Brockerhoff, E. G., H. Jactel, J. A. Parrotta, C. P. Quine, and J. Sayer. 2008. Plantation forestry and biodiversity: oxymoron or opportunity? Biodivers Conserv 17:925-951. https:/ / doi.org/10.1007/s10531-008-9380-x.

Cabaña Chávez, C. 2011. Reseña histórica de la aplicación del DL 701, de 1974, sobre fomento forestal. Corporación Nacional Forestal (CONAF), Santiago, Chile.

Camus, P. 2006. Ambiente, bosques y gestión forestal en Chile. 1541-2005. LOM Ediciones, Santiago, Chile.

Cerda, C., Barkmann, J., and R. Marggraf. 2014. Non-market economic valuation of the benefits provided by temperate ecosystems at the extreme south of the Americas. Reg Environ Change 14:1517-1531. https:/ / doi.org/10.1007/s10113014-0591-2.

Cerda, C., I. Bidegain, E. A. Silva-Rodríguez, C. Briceño, A. Promis, J. Razeto, A. Tironi-Silva, and C. L. de la Maza. 2019. Valoración social de la vida silvestre: Un estudio de caso en un hotspot de biodiversidad en Chile central. Pp. 443-471 in C. Cerda, E. A. Silva-Rodríguez and C. Briceño (eds.). Naturaleza en sociedad. Una mirada a la dimensión humana de la conservación de la biodiversidad. Ocho Libros Editores SpA, Santiago, Chile.

CONAF. 2017a. Superficie de uso de suelo regional (actualizado a agosto 2017). URL: tinyurl.com/yasyn97z.

CONAF. 2017b. Análisis del impacto de los incendios forestales ocurridos en enero y febrero de 2017 sobre los ecosistemas naturales presentes entre las regiones de Coquimbo y Los Ríos de Chile. Informe Técnico. Corporación Nacional Forestal, Santiago, Chile.

Curtis, P. S., and C. M. Gough. 2018. Forest aging, disturbance and the carbon cycle. New Phytologist 219:1188-1193. https://doi.org/10.1111/nph.15227.

Donoso, C. (ed.). 2016. Las especies arbóreas de los bosques templados de Chile y Argentina. Autoecología. Marisa Cuneo Ediciones, Valdivia, Chile.

Donoso, C., A. Promis, and R. Coopman. 2014. El clima. Pp. 25-131 in C. Donoso, M. E. González and A. Lara (eds.). Ecología Forestal. Bases para el manejo sustentable y conservación de los bosques nativos de Chile. Ediciones UACh, Valdivia, Chile.

Donoso, P., and L. A. Otero. 2005. Hacia una definición de país forestal ¿Dónde se sitúa Chile? Bosque 26:5-18. https: //doi.org/10.4067/S0717-92002005000300002.

FAO. 2011. Assessing forest degradation. Towards the development of globally applicable guidelines. Forest Resources Assessment Working Paper No. 177. FAO, Roma, Italia.

González, M. E., R. Sapiains, S. Gómez-González, R. Garreaud, A. Miranda, M. Galleguillos, M. Jacques, A. Pauchard, J. Hoyos, L. Cordero, F. Vásquez, A. Lara, P. Aldunce, V. Delgado, A. M. Arriagada Ugarte, A. Sepúlveda, L. Farías, R. García, R. J. Rondanelli, R. Ponce, F. Vargas, M. Rojas, J. P. Boisier, C. Carrasco, C. Little, M. Osses, C. Zamorano, I. Díaz-Hormazábal, A. Ceballos E. Guerra, M. Moncada, and I. Castillo. 2020. Incendios forestales en Chile: causas, impactos y resiliencia. Centro de Ciencia del Clima y la Resiliencia (CR)2, Universidad de Chile, Universidad de Concepción y Universidad Austral de Chile, Chile.

Guerra Martínez, A. 2019. US\$ 37.5 millones al año para fomento forestal. El Mercurio Campo. Consultado 24 enero 2020. URL: tinyurl.com/yd9wjpjb.

Gerding, V. 2009. La tala rasa y su efecto en la productividad de sitio. Pp. 17-39 in P. J. Donoso (ed.). Tala rasa: implicaciones y desafíos. Universidad Austral de Chile, Valdivia, Chile.

Groot, R. S., and P. J. van der Meer. 2010. Quantifying and valuing goods and services provided by plantation forests. Pp. 16-42 in J. Bauhus, P. van der Meer and M. Kanninen (eds.). Ecosystem goods and services from plantation forests. Earthscan, Londres, Gran Bretaña.

Heilmayr, R., C. Echeverría, R. Fuentes, and E. F. Lambin. 2016. A plantation-dominated forest transition in Chile. Applied Geography 75:71-82. https://doi.org/10.1016/j.apgeog.2016.07.014. 
Huber, A., A. Iroumé, and J. Bathurst. 2008. Effect of Pinus radiata plantations on water balance in Chile. Hydrol Process 22:142-148. https://doi.org/10.1002/hyp.6582.

INFOR. 2019. Anuario Forestal 2019.Boletín Estadístico No 168. Ministerio de Agricultura, Instituto Forestal, Santiago, Chile.

IPCC. 2014. 2013 revised supplementary methods and good practice guidance arising from the Kyoto Protocol. Intergovernmental Panel on Climate Change, Hayama, Japón.

Lara, A., M. E. Solari, M. del R. Prieto, and M. P. Peña. 2012. Reconstrucción de la cobertura de la vegetación y uso del suelo hacia 1550 y sus cambios a 2007 en la ecorregión de los bosques valdivianos lluviosos de Chile $\left(35^{\circ}-43^{\circ} 30^{\prime} \mathrm{S}\right)$. Bosque 33:13-23. https:/ /doi.org/10.4067/S0717-92002012000100002.

Lewis, S. L., C. E. Wheeler, E. T. A. Mitchard, and A. Koch. 2019. Restoring natural forests is the best way to remove atmospheric carbon. Nature 568:25-28. https:// doi.org/10.1038/d41586-019-01026-8.

Liao, C., Y. Luo, C. Fang, and B. Li. 2010. Ecosystem carbon stock influenced by plantation practice: Implications for planting forests as a measure of climate change mitigation. PLoS ONE 5:e10867. https://doi.org/10.1371/ journal.pone.0010867.

Lindenmayer, D. B., R. J. Hobbs, and D. Salt. 2003. Plantation forest and biodiversity conservation. Australian Forestry 66:62-66. https://doi.org/10.1080/00049158.2003.10674891.

Little, C., A. Lara, J. McPhee, and R. Urrutia. 2009. Revealing the impact of forest exotic plantations on water yield in large scale watersheds in South-Central Chile. J Hydrol 374:162-170. https://doi.org/10.1016/j.jhydrol.2009.06.011.

Little, C., J. G. Cuevas, A. Lara, M. Pino, and S. Schoenholtz. 2015. Buffer effects of streamside native forests on water provision in watersheds dominated by exotic forest plantations. Ecohydrology 8:1205-1217. https://doi.org/10.1002/ eco.1575.

Luyssaert, S., Schulze, E.-D., Börner, A., Knohl, A., Hessenmöller, D., Law, B. E., Ciais, P., and J. Grace. 2008. Old-growth forests as global carbon sinks. Nature 455:213-215. https:/ / doi.org/10.1038/nature07276.

Luebert, F., and P. Pliscoff. 2017. Sinopsis bioclimática y vegetacional de Chile. Editorial Universitaria, Santiago, Chile.

Lusk, C. H., C. Donoso, M. Jiménez, C. Moya, G. Oyarce, R. Reinoso, A. Saldaña, P. Villegas, and F. Matus. 2001. Descomposición de hojarasca de Pinus radiata y tres especies arbóreas nativas. Rev Chil Hist Nat 74:705-710. https: //doi.org/10.4067/S0716-078X2001000300016.

Odum, P. E. 1969. The strategy of ecosystem development. Science 164:262-270. https://doi.org/10.1126/ science.164.3877.262.

Oyarzún, C., Godoy, R., Staelens, J., Aracena, C., and J. Proschle. 2005. Nitrogen fluxes in a Nothofagus obliqua forest and a Pinus radiata plantation in the central valley of southern Chile. Gayana Bot 62(2):88-97. https://doi.org/10.4067/ S0717-66432005000200004.

Oyarzún, C. E., C. Frêne, G. Lacrampe, A. Huber, and P. Hervé. 2011. Propiedades hidrológicas del suelo y exportación de sedimentos en dos microcuencas de la Cordillera de la Costa en el sur de Chile con diferente cobertura vegetal. Bosque 32:10-19. https:/ / doi.org/10.4067/S0717-92002011000100002.

Parra, P. 2019. Presentación. Pages 9-10 in L. Astorga, and H. Burschel (eds.). Chile necesita un nuevo modelo forestal: Ante los desafíos climáticos, sociales y ambientales. LOM Ediciones, Santiago Chile.

Prado, J. A. 2015. Plantaciones forestales. Más allá de los árboles. Colegio de Ingenieros Forestales A.G., Santiago, Chile.

Reyes, R., C. Sepúlveda, and L. Astorga. 2014. Gobernanza del sector forestal chileno: Tensiones y conflictos entre las fuerzas de mercado y las demandas de la ciudadanía. Pp. 693-720 in C. Donoso, M. E. González and A. Lara (eds.). Ecología Forestal. Bases para el manejo sustentable y conservación de los bosques nativos de Chile. Ediciones UACh, Valdivia, Chile.

Rivas, Y., Oyarzún C., Godoy, R., and E. Valenzuela. 2009. Mineralización del nitrógeno, carbono y actividad enzimática del suelo en un bosque de Nothofagus obliqua (Mirb.) Oerst y una plantación de Pinus radiata D. Don. Del centro-sur de Chile. Rev Chil Hist Nat 82(1):119-134. https:/ / doi.org/10.4067/S0716-078X2009000100008.

Rodríguez, R., C. Marticorena, D. Alarcón, C. Baeza, L. Cavieres, V. L. Finot, N. Fuentes, A. Kiessling, M. Mihoc, A. Pauchard, E. Ruiz, P. Sánchez, and A. Marticorena. 2018. Catálogo de las plantas vasculares de Chile. Gayana Bot 75:1-430. https://doi.org/10.4067/S0717-66432018000100001.

Rodríguez García, L., Curetti, G., Garegnani, G., Grilli, G., Pastorella, F., and A. Paletto. 2016. La valoración de los servicios ecosistémicos en los ecosistemas forestales: un caso de estudio en Los Alpes Italianos. Bosque 37(1):41-52. https://doi.org/10.4067/S0717-92002016000100005.

Salas, C., P. J. Donoso, R. Vargas, C. A. Arriagada, R. Pedraza, and D. P. Soto. 2016. The forest sector in Chile: An overview and current challenges. J For 114:562-571. https://doi.org/10.5849/jof.14-062.

Schlatter, J. E., and L. Otero. 1995. Efecto de Pinus radiata sobre las características químico-nutritivas del suelo mineral superficial. Bosque 16(1):29-46. https:/ / doi.org/10.4206/bosque.1995.v16n1-03.

Schulze, E.-D., Hessenmoeller, D., Knohl, A., Luyssaert, S., Boerner, A., and J. Grace. 2009. Temperate and boreal oldgrowth forests: How do their dynamics and biodiversity differ from young stands and managed forests? Pp. 343-366 in C. Wirth, G. Gleixner and M. Heimann (eds.). Old-growth forests. Function, fate and value. Springer-Verlag, Berlín, Alemania. https://doi.org/10.1007/978-3-540-92706-8_15.

Scott, D. F., and F. W. Prinsloo. 2008. Longer-term effect of pine and eucalypt plantations on stemflow. Water Resources Research 44:W00A08. https://doi.org/10.1029/2007WR006781. 
Seidl, R., D. Thom, M. Kautz, D. Martin-Benito, M. Peltoniemi, G. Vacchiano, J. Wild, D. Ascoli, M. Petr, J. Honkaniemi, M. J. Lexer, V. Trotsiuk, P. Mairota, M. Svoboda, M. Fabrika, T. A. Nagel and C. P. O. Reyer. 2017. Forest disturbances under climate change. Nature Climate Change 7:395-402. https://doi.org/10.1038/nclimate3303.

Susaeta, E., S. Benedetti. 1990. El sector forestal y la conservación ambiental. Ambiente y Desarrollo 6(2):33-47.

Thiers, O., J. Reyes, V. Gerding, and J. E. Schlatter. 2014. Suelos en ecosistemas forestales. Pp. 133-178 in C. Donoso, M. E. González and A. Lara (eds.). Ecología Forestal. Bases para el manejo sustentable y conservación de los bosques nativos de Chile. Ediciones UACh, Valdivia, Chile.

Tomasevic, J. A, and C. F. Estades. 2008. Effects of the structure of pine plantations on their "softness" as barriers for ground-dwelling forest birds in south-central Chile. For Ecol Manag 255:810-816. https://doi.org/10.1016/ j.foreco.2007.09.073.

Úbeda, X., Sarricolea, P. 2016. Wildfires in Chile: A review. Global and Planetary Change 146:152-161. https://doi.org/ 10.1016/j.gloplacha.2016.10.004.

Vásquez-Grandón, A., P. J. Donoso, and V. Gerding. 2018. Forest degradation: When is a forest degraded? Forests 9 : 726. https://doi.org/10.3390/f9110726. 